\title{
Existence of Positive Solution for Semipositone Fractional Differential Equations Involving Riemann-Stieltjes Integral Conditions
}

\author{
Wei Wang ${ }^{1}$ and Li Huang ${ }^{2}$ \\ ${ }^{1}$ Water Transportation Planning \& Logistics Engineering Institute, College of Harbor, \\ Coastal and Offshore Engineering, Hohai University, Jiangsu, Nanjing 210098, China \\ ${ }^{2}$ National Research Center for Resettlement, Hohai University, Jiangsu, Nanjing 210098, China
}

Correspondence should be addressed to Wei Wang, 13813826667@163.com

Received 13 May 2012; Accepted 11 July 2012

Academic Editor: Yong Hong Wu

Copyright (C) 2012 W. Wang and L. Huang. This is an open access article distributed under the Creative Commons Attribution License, which permits unrestricted use, distribution, and reproduction in any medium, provided the original work is properly cited.

The existence of at least one positive solution is established for a class of semipositone fractional differential equations with Riemann-Stieltjes integral boundary condition. The technical approach is mainly based on the fixed-point theory in a cone.

\section{Introduction}

In this paper, we discuss the existence of positive solutions for the following singular semipositone fractional differential equation with nonlocal condition:

$$
\begin{gathered}
-\Phi_{\mathfrak{t}}{ }^{\alpha} x(t)=f\left(t, x(t),-\Phi_{\mathfrak{t}}^{\beta} x(t)\right)+q(t), \quad t \in(0,1), \\
\Phi_{\mathfrak{t}}^{\beta} x(0)=\Phi_{\mathfrak{t}}^{\beta+1} x(0)=0, \quad \Phi_{\mathfrak{t}}^{\beta} x(1)=\int_{0}^{1} \Phi_{\mathfrak{t}}^{\beta} x(s) d A(s),
\end{gathered}
$$

where $2<\alpha \leq 3,0<\beta<1$, and $\alpha-\beta>2, \Phi_{\mathfrak{t}}$ is the standard Riemann-Liouville derivative. $\int_{0}^{1} \Phi_{\mathfrak{t}}{ }^{\beta} x(s) d A(s)$ denotes the Riemann-Stieltjes integral, and $A$ is a function of bounded variation. $f:(0,1) \times[0,+\infty) \times(-\infty, 0] \rightarrow[0,+\infty)$ is continuous, $q:(0,1) \rightarrow(-\infty,+\infty)$ is Lebesgue integrable.

Differential equations of fractional order have been recently proved to be valuable tools in the modeling of many phenomena in various fields of science and engineering. 
Indeed, we can find numerous applications in physics, engineering (like traffic, transportation, logistics, etc.), mechanics, chemistry, and so forth, (see [1-5]). There has been a significant development in the study of fractional differential equations in recent years, see the monographs of Kilbas et al. [6], Lakshmikantham et al. [7], Podlubny [4], Samko et al. [8], the survey by Agarwal et al. [9] and some recent results [10-14].

On the other hand, the nonlocal condition given by a Riemann-Stieltjes integral is due to Webb and Infante in [15-17] and gives a unified approach to many BVPs in $[15,16]$. Motivated by [15-17], Hao et al. [18] studied the existence of positive solutions for $n$ th-order singular nonlocal boundary value problem:

$$
\begin{gathered}
x^{(n)}(t)+a(t) f(t, x(t))=0, \quad 0<t<1, \\
x^{(k)}(0)=0, \quad 0 \leq k \leq n-2, \quad x(1)=\int_{0}^{1} x(s) d A(s),
\end{gathered}
$$

where $a$ can be singular at $t=0,1, f$ also can be singular at $x=0$, but there is no singularity at $t=0,1$. The existence of positive solutions of the BVP (1.2) is obtained by means of the fixed point index theory in cones.

More recently, Zhang [19] considered the following problem whose nonlinear term and boundary condition contain integer order derivatives of unknown functions

$$
\begin{gathered}
\Phi^{\alpha} x(t)+q(t) f\left(x, x^{\prime}, \ldots, x^{(n-2)}\right)=0, \quad 0<t<1, \quad n-1<\alpha \leq n, \\
x(0)=x^{\prime}(0)=\cdots=x^{(n-2)}(0)=x^{(n-2)}(1)=0,
\end{gathered}
$$

where $\Phi^{\alpha}$ is the standard Riemann-Liouville fractional derivative of order $\alpha, q$ may be singular at $t=0$ and $f$ may be singular at $x=0, x^{\prime}=0, \ldots, x^{(n-2)}=0$. By using fixed point theorem of the mixed monotone operator, the unique existence result of positive solution to problem (1.3) was established. And then, Goodrich [20] was concerned with a partial extension of the problem (1.3) by extending boundary conditions

$$
\begin{gathered}
-\Phi^{\alpha} x(t)=f(t, x(t)), \quad 0<t<1, n-1<\alpha \leq n, \\
x^{(i)}(0)=0, \quad 0 \leq i \leq n-2, \quad \Phi^{\alpha} x(1)=0, \quad 1 \leq \alpha \leq n-2,
\end{gathered}
$$

and the author derived the Green's function for the problem (1.4) and showed that it satisfies certain properties, then by using cone theoretic techniques, a general existence theorem for (1.4) was obtained when $f(t, x)$ satisfies some growth conditions.

Recently, Rehman and Khan [21] investigated the multipoint boundary value problems for fractional differential equations of the form:

$$
\begin{aligned}
& \boldsymbol{\Phi}_{\mathfrak{t}}{ }^{\alpha} y(t)=f\left(t, y(t), \boldsymbol{\Phi}_{\mathfrak{t}}{ }^{\beta} y(t)\right), \quad t \in(0,1), \\
& y(0)=0, \quad \boldsymbol{\Phi}_{\mathfrak{t}}^{\beta} y(1)-\sum_{i=1}^{m-2} \zeta_{i} \Phi_{\mathfrak{t}}{ }^{\beta} y\left(\xi_{i}\right)=y_{0},
\end{aligned}
$$


where $1<\alpha \leq 2,0<\beta<1,0<\xi_{i}<1, \zeta_{i} \in[0,+\infty)$ with $\sum_{i=1}^{m-2} \zeta_{i} \xi_{i}^{\alpha-\beta-1}<1$. By using the Schauder fixed point theorem and the contraction mapping principle, the authors established the existence and uniqueness of nontrivial solutions for the BVP (1.5) provided that the nonlinear function $f:[0,1] \times \mathbb{R} \times \mathbb{R}$ is continuous and satisfies certain growth conditions. Since $\int_{0}^{1} \boldsymbol{\Phi}_{\mathbf{t}}^{\beta} x(s) d A(s)$ covers the multipoint BVP and integral BVP as special case, the fractional differential equations with the Riemann-Stieltjes integral condition also were extensively studied by many authors, see [22, 23]. In [23], Zhang and Han considered the existence of positive solution of the following singular fractional differential equation:

$$
\begin{aligned}
& \oplus_{\mathbf{t}}^{\alpha} x(t)+f(t, x(t))=0, \quad 0<t<1, n-1<\alpha \leq n, \\
& x^{(k)}(0)=0, \quad 0 \leq k \leq n-2, \quad x(1)=\int_{0}^{1} x(s) d A(s),
\end{aligned}
$$

where $\alpha \geq 2$ and $d A(s)$ can be a signed measure. Some growth conditions were adopted to guarantee that (1.6) has an unique positive solution, moreover, the authors also gave the iterative sequence of the solution, an error estimation, and the convergence rate of the positive solution.

Fractional differential equations like (1.1), with nonlinearities which are allowed to change sign and boundary conditions which contain nonlocal condition given by a RiemannStieltjes integral with a signed measure, are rarely studied. This type problems are referred to as semipositone problems in the literature, which arise naturally in chemical reactor theory [24]. In the recent work [25], by constructing a modified function, Zhang and Liu studied the existence of positive solution of a class of semipositone singular second-order Dirichlet boundary value problem, and when $f$ is superlinear, a sufficient condition for the existence of positive solution is obtained under the simple assumptions.

Motivated by the above work, in this paper, we establish the existence of positive solutions for the semipositone fractional differential equations (1.1) when $f$ is superlinear and involves fractional derivatives of unknown functions.

\section{Preliminaries and Lemmas}

Definition 2.1 (see [4,5]). The Riemann-Liouville fractional integral of order $\alpha>0$ of a function $x:(0,+\infty) \rightarrow \mathbb{R}$ is given by

$$
I^{\alpha} x(t)=\frac{1}{\Gamma(\alpha)} \int_{0}^{t}(t-s)^{\alpha-1} x(s) d s,
$$

provided that the right-hand side is pointwise defined on $(0,+\infty)$.

Definition 2.2 (see $[4,5]$ ). The Riemann-Liouville fractional derivative of order $\alpha>0$ of a function $x:(0,+\infty) \rightarrow \mathbb{R}$ is given by

$$
\Phi_{\mathbf{t}}^{\alpha} x(t)=\frac{1}{\Gamma(n-\alpha)}\left(\frac{d}{d t}\right)^{n} \int_{0}^{t}(t-s)^{n-\alpha-1} x(s) d s,
$$


where $n=[\alpha]+1,[\alpha]$ denotes the integer part of number $\alpha$, provided that the right-hand side is pointwise defined on $(0,+\infty)$.

Remark 2.3. If $x, y:(0,+\infty) \rightarrow \mathbb{R}$ with order $\alpha>0$, then

$$
\Phi_{\mathrm{t}}^{\alpha}(x(t)+y(t))=\Phi_{\mathrm{t}}^{\alpha} x(t)+\Phi_{\mathrm{t}}^{\alpha} y(t)
$$

Proposition 2.4 (see $[4,5])$. (1) If $x \in L^{1}(0,1), v>\sigma>0$, then

$$
I^{v} I^{\sigma} x(t)=I^{v+\sigma} x(t), \quad \Phi_{\mathrm{t}}^{\sigma} I^{v} x(t)=I^{v-\sigma} x(t), \quad \Phi_{\mathrm{t}}{ }^{\sigma} I^{\sigma} x(t)=x(t) .
$$

(2) If $v>0, \sigma>0$, then

$$
\Phi_{\mathfrak{t}} t^{\sigma-1}=\frac{\Gamma(\sigma)}{\Gamma(\sigma-v)} t^{\sigma-v-1}
$$

Proposition 2.5 (see $[4,5])$. Let $\alpha>0$, and $f(x)$ is integrable, then

$$
I^{\alpha} \boldsymbol{\Phi}_{\mathbf{t}}^{\alpha} x(t)=f(x)+c_{1} x^{\alpha-1}+c_{2} x^{\alpha-2}+\cdots+c_{n} x^{\alpha-n},
$$

where $c_{i} \in \mathbb{R}(i=1,2, \ldots, n), n$ is the smallest integer greater than or equal to $\alpha$.

Let $x(t)=I^{\beta} y(t), y(t) \in C[0,1]$, by standard discuss, one easily reduces the BVP (1.1) to the following modified problems,

$$
\begin{aligned}
& -\Phi_{\mathrm{t}}^{\alpha-\beta} y(t)=f\left(t, I^{\beta} y(t),-y(t)\right)+q(t), \\
& y(0)=y^{\prime}(0)=0, \quad y(1)=\int_{0}^{1} y(s) d A(s),
\end{aligned}
$$

and the BVP (2.7) is equivalent to the BVP (1.1).

Lemma 2.6 (see [26]). Given $y \in L^{1}(0,1)$, then the problem,

$$
\begin{aligned}
& \Phi_{\mathfrak{t}}{ }^{\alpha-\beta} x(t)+y(t)=0, \quad 0<t<1, \\
& x(0)=x^{\prime}(0)=0, \quad x(1)=0,
\end{aligned}
$$

has the unique solution

$$
x(t)=\int_{0}^{1} G(t, s) y(s) d s,
$$

where $G(t, s)$ is the Green function of the BVP (2.8) and is given by

$$
G(t, s)=\frac{1}{\Gamma(\alpha-\beta)} \begin{cases}{[t(1-s)]^{\alpha-\beta-1},} & 0 \leq t \leq s \leq 1 \\ {[t(1-s)]^{\alpha-\beta-1}-(t-s)^{\alpha-\beta-1},} & 0 \leq s \leq t \leq 1\end{cases}
$$


Lemma 2.7 (see [26]). For any $t, s \in[0,1], G(t, s)$ satisfies:

$$
\begin{aligned}
& \frac{t^{\alpha-\beta-1}(1-t) s(1-s)^{\alpha-\beta-1}}{\Gamma(\alpha-\beta)} \leq G(t, s) \\
& \quad \leq \frac{s(1-s)^{\alpha-\beta-1}}{\Gamma(\alpha-\beta-1)}, \text { or } \quad\left(\frac{t^{\alpha-\beta-1}(1-t)}{\Gamma(\alpha-\beta-1)}\right) .
\end{aligned}
$$

By Lemma 2.6, the unique solution of the problem,

$$
\begin{gathered}
\Phi_{\mathfrak{t}}^{\alpha} x(t)=0, \quad 0<t<1, \\
x(0)=x^{\prime}(0)=0, \quad x(1)=1,
\end{gathered}
$$

is $t^{\alpha-\beta-1}$. Let

$$
\mathcal{C}=\int_{0}^{1} t^{\alpha-\beta-1} d A(t), \quad B=\int_{0}^{1} t^{\alpha-\beta-1}(1-t) d A(t)
$$

and define

$$
\mathcal{G}_{A}(s)=\int_{0}^{1} G(t, s) d A(t)
$$

as in [25], one can get that the Green function for the nonlocal BVP (2.7) is given by

$$
H(t, s)=\frac{t^{\alpha-\beta-1}}{1-\mathcal{C}} \mathcal{G}_{A}(s)+G(t, s)
$$

Throughout paper one always assumes the following holds.

(H0) $A$ is a increasing function of bounded variation such that $\mathcal{G}_{A}(s) \geq 0$ for $s \in[0,1]$ and $0 \leq \mathcal{C}<1$, where $\mathcal{C}$ is defined by (2.13).

Define

$$
q_{+}(t)=\max \{q(t), 0\}, \quad q_{-}(t)=\max \{-q(t), 0\}
$$

One has the following Lemma.

Lemma 2.8. Let $1<\alpha-\beta \leq 2$ and (H0) hold, then the unique solution $w(t)$ of the linear problem,

$$
\begin{gathered}
-\Phi_{\mathrm{t}}^{\alpha-\beta} w(t)=q_{-}(t), \quad t \in(0,1), \\
w(0)=w^{\prime}(0)=0, \quad w(1)=\int_{0}^{1} w(s) d A(s),
\end{gathered}
$$


satisfies

$$
w(t) \leq \eta \gamma(t)
$$

where

$$
\eta=\frac{1}{\Gamma(\alpha-\beta-1)}\left(1+\frac{\mathcal{C}}{\mathcal{B}}\right) \int_{0}^{1} q_{-}(s) d s, \quad \gamma(t)=t^{\alpha-\beta-1}(1-t)+\frac{\mathcal{B}}{1-\mathcal{C}} t^{\alpha-\beta-1}
$$

Proof. By (2.11) and that $A(t)$ is a increasing function of bounded variation, we have

$$
\mathcal{G}_{A}(s)=\int_{0}^{1} G(t, s) d A(t) \leq \int_{0}^{1} \frac{t^{\alpha-\beta-1}}{\Gamma(\alpha-\beta-1)} d A(t)=\frac{\mathcal{C}}{\Gamma(\alpha-\beta-1)} .
$$

Consequently,

$$
\begin{aligned}
w(t) & =\int_{0}^{1} H(t, s) q_{-}(t) d s=\int_{0}^{1}\left(\frac{t^{\alpha-\beta-1}}{1-\mathcal{C}} \mathcal{G}_{A}(s)+G(t, s)\right) q_{-}(s) d s \\
& \leq \frac{t^{\alpha-\beta-1}(1-t)}{\Gamma(\alpha-\beta-1)} \int_{0}^{1} q_{-}(s) d s+\frac{\mathcal{C} t^{\alpha-\beta-1}}{\Gamma(\alpha-\beta-1)(1-\mathcal{C})} \int_{0}^{1} q_{-}(s) d s \\
& \leq \frac{1}{\Gamma(\alpha-\beta-1)}\left(1+\frac{\mathcal{C}}{B}\right)\left(t^{\alpha-\beta-1}(1-t)+\frac{B}{1-\mathcal{C}} t^{\alpha-\beta-1}\right) \int_{0}^{1} q_{-}(s) d s \\
& =\eta \gamma(t) .
\end{aligned}
$$

Remark 2.9. (1) $\gamma(t)$ satisfies

$$
\frac{(1-\mathcal{C}) \gamma(t)}{2} \leq \frac{(1-\mathcal{C}) \gamma(t)}{\alpha-\beta-1} \leq 1
$$

(2)

$$
H(t, s) \leq \frac{\mathcal{G}_{A}(s)}{1-\mathcal{C}}+\frac{s(1-s)^{\alpha-\beta-1}}{\Gamma(\alpha-\beta-1)}=\Phi(s)
$$

In fact, since $1<\alpha-\beta-1<2$, the left side of (1) clearly holds. For right side of (1), from $\mathbb{B} \leq \mathcal{C}$, one gets

$$
\gamma(t) \leq 1+\frac{B}{1-\mathcal{C}} \leq 1+\frac{\mathcal{C}}{1-\mathcal{C}}=\frac{1}{1-\mathcal{C}^{\prime}}
$$


thus we have

$$
\frac{(1-\mathcal{C}) \gamma(t)}{\alpha-\beta-1} \leq 1
$$

(2) is obvious from (2.11).

Now define a function $[\cdot]^{*}$ for any $z \in C[0,1]$ by

$$
[z(t)]^{*}= \begin{cases}z(t), & z(t) \geq 0 \\ 0, & z(t)<0\end{cases}
$$

and consider the following approximate problem of the BVP (2.7):

$$
\begin{aligned}
-\Phi_{\mathbf{t}}{ }^{\alpha-\beta} v(t) & =f\left(t, I^{\beta}[v(t)-w(t)]^{*},-[v(t)-w(t)]^{*}\right)+q_{+}(t), \\
v(0) & =v^{\prime}(0)=0, \quad v(1)=\int_{0}^{1} v(s) d A(s) .
\end{aligned}
$$

Lemma 2.10. Suppose $v$ is a positive solution of the problem (2.27) and satisfies $v(t) \geq w(t), t \in$ $[0,1]$, then $v-w$ is a positive solution of the problem $(2.7)$, consequently, $I^{\beta}[v(t)-w(t)]$ also is a positive solution of the BVP (1.1).

Proof. In fact, if $v$ is a positive solution of the BVP (2.27) such that $v(t) \geq w(t)$ for any $t \in[0,1]$, then, from (2.27) and the definition of $[z(t)]^{*}$, we have

$$
\begin{aligned}
-\Phi_{\mathfrak{t}}^{\alpha-\beta} v(t) & =f\left(t, I^{\beta}(v(t)-w(t)),-(v(t)-w(t))\right)+q_{+}(t), \\
v(0) & =v^{\prime}(0)=0, \quad v(1)=\int_{0}^{1} v(s) d A(s) .
\end{aligned}
$$

Let $y=v-w$, then we have

$$
w(0)=w^{\prime}(0)=0, \quad w(1)=\int_{0}^{1} w(s) d A(s),
$$

and $\Phi_{\mathrm{t}}^{\alpha-\beta} y(t)=\Phi_{\mathrm{t}}^{\alpha-\beta} v(t)-\Phi_{\mathrm{t}}^{\alpha-\beta} w(t)$, which implies that

$$
-\Phi_{\mathrm{t}}^{\alpha-\beta} v(t)=-\Phi_{\mathrm{t}}^{\alpha-\beta} y(t)+q_{-}(t)
$$

Since $q(t)=q_{+}(t)-q_{-}(t)$, then $(2.27)$ is transformed to (2.7), that is, $v-w$ is a positive solution of the BVP (2.27). By $(2.7), I^{\beta}[v(t)-w(t)]$ is a positive solution of the BVP (1.1). 
It is well known that the BVP (2.27) is equivalent to the fixed points of the mapping $T$ given by

$$
(T v)(t)=\int_{0}^{1} H(t, s)\left(f\left(s, I^{\beta}[v(s)-w(s)]^{*},-[v(s)-w(s)]^{*}\right)+q_{+}(s)\right) d s
$$

The basic space used in this paper is $E=C([0,1] ; \mathbb{R})$, where $\mathbb{R}$ is a real number set. Obviously, the space $E$ is a Banach space if it is endowed with the norm as follows:

$$
\|v\|=\max _{t \in[0,1]}|v(t)|
$$

for any $u \in E$. Let

$$
P=\left\{v \in E: v(t) \geq \frac{(1-\mathcal{C}) \gamma(t)}{4}\|v\|\right\}
$$

then $P$ is a cone of $E$.

For the convenience in presentation, we now present some assumptions to be used in the rest of the paper.

(H1) For any fixed $t \in(0,1)$ and for any $c \in(0,1]$, there exist constants $\mu_{i}>1, i=$ $1,2,3,4$, such that, for all $(t, u, v) \in(0,1) \times[0,+\infty) \times(-\infty,+0]$,

$$
\begin{gathered}
c^{\mu_{1}} f(t, u, v) \leq f(t, c u, v) \leq c^{\mu_{3}} f(t, u, v), \\
c^{\mu_{2}} f(t, u, v) \leq f(t, u, c v) \leq c^{\mu_{4}} f(t, u, v) . \\
\left(\text { H2) } \int_{0}^{1} q_{-}(s) d s>0, \text { for any } s \in(0,1), f(s, 1,-1)>0,\right. \text { and } \\
\int_{0}^{1} \Phi(s)\left[f(s, 1,-1)+q_{+}(s)\right] d s \leq \frac{r}{2(1+r)^{\mu_{1}+\mu_{2}}},
\end{gathered}
$$

where

$$
r=\frac{8 \eta}{1-\mathcal{C}^{\prime}}
$$

and $\mathcal{C}, \eta, \Phi$ are defined by (2.13), (2.19), and (2.23), respectively.

Remark 2.11. If $c>1$, the reversed inequality of (2.34) holds, that is, for all $(t, u, v) \in(0,1) \times$ $[0,+\infty) \times(-\infty,+0]$, one has

$$
c^{\mu_{3}} f(t, u, v) \leq f(t, c u, v) \leq c^{\mu_{1}} f(t, u, v), c^{\mu_{4}} f(t, u, v) \leq f(t, u, c v) \leq c^{\mu_{2}} f(t, u, v)
$$


Lemma 2.12. Suppose (H1)-(H2) holds, for any fixed $t \in(0,1), f(t, u, v)$ is nondecreasing in $u$ on $[0,+\infty)$ and nonincreasing in $v$ on $(-\infty, 0]$; and for any $[a, b] \subset(0,1)$,

$$
\lim _{(u, v) \rightarrow(+\infty,-\infty)} \min _{t \in[a, b]} \frac{f(t, u, v)}{|u||v|}=+\infty
$$

Proof. Let $0 \leq u_{1} \leq u_{2}$. If $u_{2}=0$, obviously $f\left(t, u_{1}, v\right) \leq f\left(t, u_{2}, v\right)$ holds. If $u_{2} \neq 0$, let $c_{0}=u_{1} / u_{2}$, then $0<c_{0} \leq 1$. It follows from (H1) that

$$
f\left(t, u_{1}, v\right)=f\left(t, c_{0} u_{2}, v\right) \leq c_{0}^{\mu_{3}} f\left(t, u_{2}, v\right) \leq f\left(t, u_{2}, v\right)
$$

Thus $f(t, u, v)$ is nondecreasing in $u$ on $[0, \infty)$.

On the other hand, for any $v_{1} \leq v_{2} \leq 0$, if $v_{1}=0$, obviously $f\left(t, u, v_{1}\right) \geq f\left(t, u, v_{2}\right)$ holds. If $v_{1} \neq 0$, let $c_{1}=v_{2} / v_{1}$, then $0<c_{1} \leq 1$. By (H1), we have

$$
f\left(t, u, v_{2}\right)=f\left(t, u, c_{1} v_{1}\right) \leq c_{1}^{\mu_{4}} f\left(t, u, v_{1}\right) \leq f\left(t, u, v_{1}\right)
$$

Thus $f(t, u, v)$ is nonincreasing in $v$ on $(-\infty, 0]$.

Now choose $u>1$ and $v<-1$, then by Remark 2.11, we have

$$
f(t, u, v)=f(t,|u|,-|v|) \geq|u|^{\mu_{3}} f(t, 1,-|v|) \geq|u|^{\mu_{3}}|v|^{\mu_{4}} f(t, 1,-1) .
$$

Thus for any $[a, b] \subset(0,1)$, and any $t \in[a, b]$, we have

$$
\min _{t \in[a, b]} \frac{f(t, u, v)}{|u||v|} \geq|u|^{\mu_{3}-1}|v|^{\mu_{4}-1} \min _{t \in[a, b]} f(t, 1,-1)>0 .
$$

Therefore

$$
\lim _{(u, v) \rightarrow(+\infty,-\infty)} \min _{t \in[a, b]} \frac{f(t, u, v)}{|u||v|}=+\infty .
$$

Lemma 2.13. Assume that (H0)-(H2) holds. Then $T: P \rightarrow P$ is well defined. Furthermore, $T$ : $P \rightarrow P$ is a completely continuous operator.

Proof. For any fixed $v \in P$, there exists a constant $L>0$ such that $\|v\| \leq L$. And then,

$$
\begin{gathered}
0 \leq[v(s)-w(s)]^{*} \leq v(s) \leq\|v\| \leq L, \\
0 \leq I^{\beta}[v(s)-w(s)]^{*}=\int_{0}^{t} \frac{(t-s)^{\beta-1}[v(s)-w(s)]^{*}}{\Gamma(\beta)} d s \leq \frac{L}{\Gamma(\beta)} .
\end{gathered}
$$


By (2.44) and (H1)-(H2), we have

$$
\begin{aligned}
(T v)(t) & =\int_{0}^{1} H(t, s)\left(f\left(s, I^{\beta}[v(s)-w(s)]^{*},-[v(s)-w(s)]^{*}\right)+q_{+}(s)\right) d s \\
& \leq \int_{0}^{1} H(t, s)\left(f\left(s, \frac{L}{\Gamma(\beta)}+1,-(L+1)\right)+q_{+}(s)\right) d s \\
& \leq\left(\frac{L}{\Gamma(\beta)}+1\right)^{\mu_{1}}(L+1)^{\mu_{2}} \int_{0}^{1} \Phi(s)\left[f(s, 1,-1)+q_{+}(s)\right] d s<+\infty
\end{aligned}
$$

which implies that the operator $T: P \rightarrow E$ is well defined.

Next let $f^{*}(s)=f\left(s, I^{\beta}[v(s)-w(s)]^{*},-[v(s)-w(s)]^{*}\right)+q_{+}(s)$, for any $v \in P$, by $(2.11)$, we have

$$
\begin{aligned}
\|T v\| & \leq \int_{0}^{1} \frac{s(1-s)^{\alpha-\beta-1}}{\Gamma(\alpha-\beta-1)} f^{*}(s) d s+\frac{t^{\alpha-\beta-1}}{1-\mathcal{C}} \int_{0}^{1} \mathcal{G}_{A}(s) f^{*}(s) d s \\
& \leq \frac{1}{1-\mathcal{C}} \int_{0}^{1} \frac{s(1-s)^{\alpha-\beta-1}}{\Gamma(\alpha-\beta-1)} f^{*}(s) d s+\frac{t^{\alpha-\beta-1}}{1-\mathcal{C}} \int_{0}^{1} \mathcal{G}_{A}(s) f^{*}(s) d s .
\end{aligned}
$$

On the other hand, by (2.11), (2.22), and (2.46), we also have

$$
\begin{aligned}
(T u)(t) \geq & t^{\alpha-\beta-1}(1-t) \int_{0}^{1} \frac{s(1-s)^{\alpha-\beta-1}}{\Gamma(\alpha-\beta)} f^{*}(s) d s+\frac{t^{\alpha-\beta-1}}{1-\mathcal{C}} \int_{0}^{1} \mathcal{G}_{A}(s) f^{*}(s) d s \\
\geq & \frac{1}{2} t^{\alpha-\beta-1}(1-t) \int_{0}^{1} \frac{s(1-s)^{\alpha-\beta-1}}{\Gamma(\alpha-\beta)} f^{*}(s) d s+\frac{t^{\alpha-\beta-1}}{1-\mathcal{C}} \int_{0}^{1} \mathcal{G}_{A}(s) f^{*}(s) d s \\
= & \frac{1}{2}\left\{t^{\alpha-\beta-1}(1-t) \int_{0}^{1} \frac{s(1-s)^{\alpha-\beta-1}}{\Gamma(\alpha-\beta)} f^{*}(s) d s+\frac{t^{\alpha-\beta-1}}{1-\mathcal{C}} \int_{0}^{1} \mathcal{G}_{A}(s) f^{*}(s) d s\right\} \\
& +\frac{t^{\alpha-\beta-1}}{2(1-\mathcal{C})} \int_{0}^{1} \mathcal{G}_{A}(s) f^{*}(s) d s \\
\geq & \frac{1}{2}\left\{t^{\alpha-\beta-1}(1-t) \int_{0}^{1} \frac{s(1-s)^{\alpha-\beta-1}}{\Gamma(\alpha-\beta)} f^{*}(s) d s+\frac{B t^{\alpha-\beta-1}}{1-\mathcal{C}} \int_{0}^{1} \frac{s(1-s)^{\alpha-\beta-1}}{\Gamma(\alpha-\beta)} f^{*}(s) d s\right\} \\
& +\frac{t^{\alpha-\beta-1}}{2(1-\mathcal{C})} \int_{0}^{1} \mathcal{G}_{A}(s) f^{*}(s) d s
\end{aligned}
$$




$$
\begin{aligned}
& =\frac{(1-\mathcal{C}) \gamma(t)}{2(\alpha-\beta-1)} \times \frac{1}{1-\mathcal{C}} \int_{0}^{1} \frac{s(1-s)^{\alpha-\beta-1}}{\Gamma(\alpha-\beta-1)} f^{*}(s) d s+\frac{t^{\alpha-\beta-1}}{2(1-\mathcal{C})} \int_{0}^{1} \mathcal{G}_{A}(s) f^{*}(s) d s \\
& \geq \frac{(1-\mathcal{C}) \gamma(t)}{2(\alpha-\beta-1)}\left\{\frac{1}{1-\mathcal{C}} \int_{0}^{1} \frac{s(1-s)^{\alpha-\beta-1}}{\Gamma(\alpha-\beta-1)} f^{*}(s) d s+\frac{t^{\alpha-\beta-1}}{1-\mathcal{C}} \int_{0}^{1} \mathcal{G}_{A}(s) f^{*}(s) d s\right\} \\
& \geq \frac{(1-\mathcal{C}) \gamma(t)}{4}\left\{\frac{1}{1-\mathcal{C}} \int_{0}^{1} \frac{s(1-s)^{\alpha-\beta-1}}{\Gamma(\alpha-\beta-1)} f^{*}(s) d s+\frac{t^{\alpha-\beta-1}}{1-\mathcal{C}} \int_{0}^{1} \mathcal{G}_{A}(s) f^{*}(s) d s\right\} .
\end{aligned}
$$

So we have

$$
(T v)(t) \geq \frac{(1-\mathcal{C}) \gamma(t)}{4}\|T v\|, \quad t \in[0,1]
$$

which yields that $T(P) \subset P$.

At the end, using standard arguments, according to the Ascoli-Arzela Theorem, one can show that $T: P \rightarrow P$ is continuous. Thus $T: P \rightarrow P$ is a completely continuous operator.

Lemma 2.14 (see [27]). Let $E$ be a real Banach space, $P \subset E$ be a cone. Assume $\Omega_{1}, \Omega_{2}$ are two bounded open subsets of $E$ with $\theta \in \Omega_{1}, \bar{\Omega}_{1} \subset \Omega_{2}$, and let $T: P \cap\left(\bar{\Omega}_{2} \backslash \Omega_{1}\right) \rightarrow P$ be a completely continuous operator such that either

(1) $\|T x\| \leq\|x\|, x \in P \cap \partial \Omega_{1}$, and $\|T x\| \geq\|x\|, x \in P \cap \partial \Omega_{2}$ or

(2) $\|T x\| \geq\|x\|, x \in P \cap \partial \Omega_{1}$, and $\|T x\| \leq\|x\|, x \in P \cap \partial \Omega_{2}$.

Then $T$ has a fixed point in $P \cap\left(\bar{\Omega}_{2} \backslash \Omega_{1}\right)$.

\section{Main Results}

Theorem 3.1. Suppose (H0)-(H2) hold. Then the BVP (1.1) has at least one positive solutions $x(t)$, and $x(t)$ satisfies

$$
x(t) \geq \frac{\eta \Gamma(\beta) \Gamma(\alpha-\beta)}{\Gamma(\alpha)}\left[\left(1+\frac{B}{1-\mathcal{C}}\right) t^{\alpha-1}-\frac{\alpha-\beta}{\alpha} t^{\alpha}\right], \quad t \in[0,1] .
$$

Proof. Let

$$
r=\frac{8 \eta}{1-\mathcal{C}^{\prime}}
$$

and $\Omega_{1}=\{v \in P:\|v\|<r\}$. Then, for any $v \in \partial \Omega_{1}, s \in[0,1]$, notice that $0<\beta<1$, we have

$$
\begin{gathered}
{[v(s)-w(s)]^{*} \leq v(s) \leq\|v\| \leq r,} \\
I^{\beta}[v(s)-w(s)]^{*}=\frac{1}{\Gamma(\beta)} \int_{0}^{s}(s-t)^{\beta-1}[v(t)-w(t)]^{*} d t \leq \frac{r}{\Gamma(\beta)} \leq r .
\end{gathered}
$$


By (H1) and (H2), one has

$$
\begin{aligned}
\|T v\| & =\max _{0 \leq t \leq 1} \int_{0}^{1} H(t, s)\left[f\left(s, I^{\beta}[v(s)-w(s)]^{*},-[v(s)-w(s)]^{*}\right)+q_{+}(s)\right] d s \\
& \leq \int_{0}^{1} \Phi(s)\left[f\left(s, \frac{r}{\Gamma(\beta)},-r\right)+q_{+}(s)\right] d s \\
& \leq \int_{0}^{1} \Phi(s)\left[f(s, r+1,-(r+1))+q_{+}(s)\right] d s \\
& \leq 2(r+1)^{\mu_{1}+\mu_{2}} \int_{0}^{1} \Phi(s)\left[f(s, 1,-1)+q_{+}(s)\right] d s \\
& \leq r=\|v\| .
\end{aligned}
$$

Therefore,

$$
\|T v\| \leq\|v\|, \quad v \in P \cap \partial \Omega_{1} .
$$

On the other hand, choose a real number $M>0$ such that

$$
\frac{a^{\alpha-\beta}(1-b)^{\alpha-\beta}}{\Gamma(\alpha-\beta)} \times \frac{M(1-\mathcal{C})^{2} a^{\beta}}{64 \Gamma(\beta+1)}\left(a^{\alpha-\beta-1}(1-b)+\frac{B}{1-\mathcal{C}} a^{\alpha-\beta-1}\right)^{2} \geq 1
$$

From (2.38), there exists $N>r$ such that, for any $t \in[a, b]$,

$$
f(t, u, v) \geq M|u||v|, \quad|u|,|v| \geq N
$$

Take

$R$

$$
>\max \left\{1, \frac{8 N}{(1-\mathcal{C})\left(a^{\alpha-\beta-1}(1-b)+\frac{B}{1-\mathcal{C}} a^{\alpha-\beta-1}\right)}, \frac{8 N \Gamma(\beta+1)}{(1-\mathcal{C}) a^{\beta}\left(a^{\alpha-\beta-1}(1-b)+\frac{B}{1-\mathcal{C}} a^{\alpha-\beta-1}\right)}, r\right\},
$$


then $R>r$. Let $\Omega_{2}=\{v \in P:\|v\|<R\}$, for any $v \in P \cap \partial \Omega_{2}$ and for any $t \in[a, b]$, we have

$$
\begin{aligned}
v(t)-w(t) & \geq v(t)-\eta \gamma(t) \geq v(t)-\frac{4}{(1-\mathcal{C}) R} v(t) \geq \frac{1}{2} v(t) \\
& \geq \frac{(1-\mathcal{C}) \gamma(t)}{8} R \geq \frac{(1-\mathcal{C})}{8}\left(a^{\alpha-\beta-1}(1-b)+\frac{B}{1-\mathcal{C}} a^{\alpha-\beta-1}\right) R \geq N>0, \\
I^{\beta}[v(t)-w(t)] & \geq \frac{(1-\mathcal{C})}{8}\left(a^{\alpha-\beta-1}(1-b)+\frac{B}{1-\mathcal{C}} a^{\alpha-\beta-1}\right) R \int_{0}^{t} \frac{(t-s)^{\beta-1}}{\Gamma(\beta)} d s \\
& \geq \frac{(1-\mathcal{C}) a^{\beta}}{8 \Gamma(\beta+1)}\left(a^{\alpha-\beta-1}(1-b)+\frac{B}{1-\mathcal{C}} a^{\alpha-\beta-1}\right) R \geq N>0 .
\end{aligned}
$$

So for any $v \in P \cap \partial \Omega_{2}, t \in[a, b]$, by (3.7)-(3.9), we have

$$
\begin{aligned}
\|T v\| & \geq \int_{0}^{1} H(t, s)\left(f\left(s, I^{\beta}[v(s)-w(s)]^{*},-[v(s)-w(s)]^{*}\right)+q_{+}(s)\right) d s \\
& \geq \int_{0}^{1} G(t, s) f\left(s, I^{\beta}[v(s)-w(s)]^{*},-[v(s)-w(s)]^{*}\right) d s s \\
& \geq \frac{t^{\alpha-\beta-1}(1-t)}{\Gamma(\alpha-\beta)} \int_{a}^{b} s(1-s)^{\alpha-\beta-1} f\left(s, I^{\beta}[v(s)-w(s)]^{*},-[v(s)-w(s)]^{*}\right) d s \\
& \geq \frac{a^{\alpha-\beta-1}(1-b)}{\Gamma(\alpha-\beta)} \int_{a}^{b} s(1-s)^{\alpha-\beta-1} M\left|I^{\beta}[v(s)-w(s)]\right| v(s)-w(s) \mid d s \\
& \geq \frac{a^{\alpha-\beta}(1-b)^{\alpha-\beta}}{\Gamma(\alpha-\beta)} \times \frac{M(1-\mathcal{C})^{2} a^{\beta}}{64 \Gamma(\beta+1)}\left(a^{\alpha-\beta-1}(1-b)+\frac{B}{1-\mathcal{C}} a^{\alpha-\beta-1}\right)^{2} R^{2} \\
& \geq R^{2} \geq R=\|x\| .
\end{aligned}
$$

Thus

$$
\|T v\| \geq\|v\|, \quad v \in P \cap \partial \Omega_{2} .
$$

By Lemma 2.14, $T$ has at least one fixed points $v$ such that $r \leq\|v\| \leq R$. In the end,

$$
\begin{aligned}
v(t)-w(t) & \geq \frac{(1-\mathcal{C}) \gamma(t)}{4}\|v\|-\eta \gamma(t) \geq \frac{(1-\mathcal{C}) \gamma(t)}{4} r-\eta \gamma(t) \\
& \geq \eta \gamma(t)>0, \quad t \in(0,1)
\end{aligned}
$$


Equation (3.12) implies that $v(t)>w(t), t \in(0,1)$, and

$$
\begin{aligned}
x(t) & =I^{\beta}(v(t)-w(t)) \geq \eta I^{\beta} \gamma(t)=\eta \int_{0}^{t}(t-s)^{\beta-1} \gamma(s) d s \\
& =\frac{\eta \Gamma(\beta) \Gamma(\alpha-\beta)}{\Gamma(\alpha)}\left[\left(1+\frac{B}{1-\mathcal{C}}\right) t^{\alpha-1}-\frac{\alpha-\beta}{\alpha} t^{\alpha}\right]>0, \quad t \in(0,1) .
\end{aligned}
$$

So by Lemma 2.10, the BVP (1.1) has at least one positive solution $x$, and $x$ satisfies (3.13).

Example 3.2. Consider the following semipositone boundary value problem with fractional order $\alpha=21 / 8$ :

$$
\begin{gathered}
-\Phi^{21 / 8} x(t)=\frac{x^{2}(t)+\left(\Phi^{1 / 8} x(t)\right)^{3}}{10^{12} \times \sqrt[4]{(1-t)}}-\frac{1}{\sqrt{t}}, \quad 0<t<1, \\
\boldsymbol{\Phi}^{1 / 8} x(0)=\boldsymbol{\Phi}^{9 / 8} x(0)=0, \quad \boldsymbol{\Phi}^{1 / 8} x(1)=\int_{0}^{1} \boldsymbol{\Phi}^{1 / 8} x(s) d A(s) .
\end{gathered}
$$

Let

$$
A(t)=\left\{\begin{array}{c}
0, t \in\left[0, \frac{1}{4}\right), \\
1, t \in\left[\frac{1}{4}, \frac{3}{4}\right), \\
2, t \in\left[\frac{3}{4}, 1\right],
\end{array}\right.
$$

then the BVP (3.14) becomes the 4-Point BVP with coefficients

$$
\begin{aligned}
-\Phi^{21 / 8} x(t) & =\frac{x^{2}(t)+\left(\boldsymbol{\Phi}^{1 / 8} x(t)\right)^{3}}{10^{12} \times \sqrt[4]{(1-t)}-\frac{1}{\sqrt{t}}, \quad 0<t<1,} \\
\boldsymbol{\Phi}^{1 / 8} x(0) & =\boldsymbol{\Phi}^{9 / 8} x(0)=0, \quad \boldsymbol{\Phi}^{1 / 8} x(1)=\boldsymbol{\Phi}^{1 / 8} x\left(\frac{1}{4}\right)+\boldsymbol{\Phi}^{1 / 8} x\left(\frac{3}{4}\right) .
\end{aligned}
$$

Then the BVP (3.14) has at least one positive solution $x(t)$, and $x(t)$ satisfies

$$
x(t) \geq 62.4289\left[1.1357 t^{5 / 2}-\frac{10}{21} t^{21 / 8}\right], \quad t \in[0,1]
$$


Proof. Obviously, $\alpha=21 / 8, \beta=1 / 8$, and

$$
\begin{aligned}
& 0 \leq \mathcal{C}=\int_{0}^{1} t^{3 / 2} d A(t)=\left(\frac{1}{4}\right)^{3 / 2}+\left(\frac{3}{4}\right)^{3 / 2} \approx 0.7745<1 \\
& B=\int_{0}^{1} t^{3 / 2}(1-t) d A(t)=\frac{3}{4}\left(\frac{1}{4}\right)^{3 / 2}+\frac{1}{4}\left(\frac{3}{4}\right)^{3 / 2} \approx 0.2561
\end{aligned}
$$

On the other hand, we have

$$
\begin{gathered}
G(t, s)= \begin{cases}G_{1}(t, s)=\frac{[t(1-s)]^{3 / 2}}{\Gamma(5 / 2)}, & 0 \leq t \leq s \leq 1, \\
G_{2}(t, s)=\frac{[t(1-s)]^{3 / 2}-(t-s)^{3 / 2}}{\Gamma(5 / 2)}, & 0 \leq s \leq t \leq 1,\end{cases} \\
\mathcal{G}_{A}(s)= \begin{cases}G_{2}\left(\frac{1}{4}, s\right)+2 G_{2}\left(\frac{3}{4}, s\right), & 0 \leq s<\frac{1}{4}, \\
G_{1}\left(\frac{1}{4}, s\right)+2 G_{2}\left(\frac{3}{4}, s\right), & \frac{1}{4} \leq s<\frac{3}{4}, \\
G_{1}\left(\frac{1}{4}, s\right)+2 G_{1}\left(\frac{3}{4}, s\right), & \frac{3}{4} \leq s \leq 1 .\end{cases}
\end{gathered}
$$

Thus $\mathcal{G}_{A}(s) \geq 0,0 \leq \mathcal{C}<1$ and $A(s)$ is increasing. So (H0) holds.

Take

$$
f(t, u, v)=\frac{u^{2}-v^{3}}{10^{12} \times \sqrt[4]{(1-t)}}, \quad(t, u, v) \in(0,1) \times[0,+\infty) \times(-\infty, 0], \quad q(t)=-\frac{1}{\sqrt{t}^{\prime}}
$$

then

$$
f\left(t, x(t),-\Phi_{\mathbf{t}}^{1 / 8} x(t)\right)=\frac{x^{2}(t)+\left(\Phi^{1 / 8} x(t)\right)^{3}}{10^{12} \times \sqrt[4]{(1-t)}}, \quad q_{+}(t)=0, \quad q_{-}(t)=\frac{1}{\sqrt{t}}
$$

For any $0<c \leq 1$, we also have

$$
\begin{aligned}
& c^{3} f(t, u, v) \leq f(t, c u, v) \leq c^{3 / 2} f(t, u, v) \\
& c^{2} f(t, u, v) \leq f(t, c u, v) \leq c^{4} f(t, u, v)
\end{aligned}
$$

Then (H1) holds. 
In the end, we notice

$$
\begin{aligned}
\Phi(s) & =\frac{\mathcal{G}_{A}(s)}{1-\mathcal{C}}+\frac{s(1-s)^{\alpha-\beta-1}}{\Gamma(\alpha-\beta-1)} \leq \frac{\mathcal{C}}{\Gamma(\alpha-\beta-1)(1-\mathcal{C})}+\frac{1}{\Gamma(\alpha-\beta-1)} \\
& =\frac{1}{\Gamma(\alpha-\beta-1)(1-\mathcal{C})} \approx 5.0041,
\end{aligned}
$$

then

$$
\int_{0}^{1} \Phi(s)\left[f(s, 1,-1)+q_{+}(s)\right] d s \leq 5.0041 \int_{0}^{1} \frac{2}{10^{12} \times \sqrt[4]{(1-t)}} d s \approx 1.33443 \times 10^{11}
$$

Moreover,

$$
\begin{gathered}
\eta=\frac{1}{\Gamma(\alpha-\beta-1)}\left(1+\frac{\mathcal{C}}{B}\right) \int_{0}^{1} q_{-}(s) d s \approx 9.0817, \quad r=\frac{8 \eta}{1-\mathcal{C}}=322.1889, \\
\frac{r}{2(1+r)^{\mu_{1}+\mu_{2}}}=\frac{322.1889}{2(1+322.1889)^{5}} \approx 4.5688 \times 10^{-11}
\end{gathered}
$$

which implies that (H2) holds.

According to Theorem 3.1, the BVP (3.14) has at least one positive solution $x(t)$, and $x(t)$ satisfies

$$
x(t) \geq 62.4289\left[1.1357 t^{5 / 2}-\frac{10}{21} t^{21 / 8}\right], \quad t \in[0,1]
$$

\section{Acknowledgments}

The paper is Funded by the Humanities and Social Sciences Foundation of Ministry of Education (no. 09YJC630056), the National Natural Science Foundation of China (no. 51009060, no. 50909042), the Fundamental Research Funds for the Central Universities (no. 2009B13414), and the Priority Academic Program Development of Jiangsu Higher Education Institutions(Coastal Development Conservancy).

\section{References}

[1] W. G. Glockle and T. F. Nonnenmacher, "A fractional calculus approach of self-similar protein dynamics," Biophysical Journal, vol. 68, no. 1, pp. 46-53, 1995.

[2] R. Hilfer, Applications of Fractional Calculus in Physics, World Scientific, River Edge, NJ, USA, 2000.

[3] F. Metzler, W. Schick, H. G. Kilian, and T. F. Nonnenmache, "Relaxation in filled polymers: a fractional calculus approach," Journal of Chemical Physics, vol. 103, no. 16, pp. 7180-7186, 1995.

[4] I. Podlubny, Fractional Differential Equations, Mathematics in Science and Engineering, Academic Press, London, UK, 1999. 
[5] I. Podlubny, "Geometric and physical interpretation of fractional integration and fractional differentiation," Fractional Calculus \& Applied Analysis, vol. 5, no. 4, pp. 367-386, 2002.

[6] A. A. Kilbas, H. M. Srivastava, and J. J. Trujillo, Theory and Applications of Fractional Differential Equations, vol. 204, Elsevier Science B.V., Amsterdam, The Netherlands, 2006.

[7] V. Lakshmikantham, S. Leela, and J. Vasundhara, Theory of Fractional Dynamic Systems, Cambridge Academic Publishers, Cambridge, UK, 2009.

[8] S. G. Samko, A. A. Kilbas, and O. I. Marichev, Fractional Integrals and derivatives Theory and Applications, Gordon and Breach Science Publishers, Yverdon-les-Bains, Switzerland, 1993.

[9] R. P. Agarwal, M. Benchohra, and S. Hamani, "A survey on existence results for boundary value problems of nonlinear fractional differential equations and inclusions," Acta Applicandae Mathematicae, vol. 109, no. 3, pp. 973-1033, 2010.

[10] X. Zhang, L. Liu, and Y. Wu, "The eigenvalue problem for a singular higher order fractional differential equation involving fractional derivatives," Applied Mathematics and Computation, vol. 218, no. 17, pp. 8526-8536, 2012.

[11] X. Zhang, L. Liu, B. Wiwatanapataphee, and Y. Wu, "Positive solutions of eigenvalue problems for a class of fractional differential equations with derivatives," Abstract and Applied Analysis, vol. 2012, Article ID Article ID 512127, 16 pages, 2012.

[12] X. Zhang, L. Liu, and Y. Wu, "Multiple positive solutions of a singular fractional differential equation with negatively perturbed term," Mathematical and Computer Modelling, vol. 55, no. 3-4, pp. 1263-1274, 2012.

[13] Y. Wang, L. Liu, and Y. Wu, "Positive solutions of a fractional boundary value problem with changing sign nonlinearity," Abstract and Applied Analysis, vol. 2012, Article ID 149849, 12 pages, 2012.

[14] X. Zhang, L. Liu, and Y. Wu, "Existence results for multiple positive solutions of nonlinear higher orderperturbed fractional differential equations with derivatives," Applied Mathematics and Computation, http:/ /dx.doi.org/10.1016/j.amc.2012.07.046.

[15] J. R. L. Webb and G. Infante, "Positive solutions of nonlocal boundary value problems involving integral conditions," Nonlinear Differential Equations and Applications, vol. 15, no. 1-2, pp. 45-67, 2008.

[16] J. R. L. Webb and G. Infante, "Positive solutions of nonlocal boundary value problems: a unified approach," Journal of the London Mathematical Society, vol. 74, no. 3, pp. 673-693, 2006.

[17] J. R. L. Webb, "Nonlocal conjugate type boundary value problems of higher order," Nonlinear Analysis. Theory, Methods \& Applications, vol. 71, no. 5-6, pp. 1933-1940, 2009.

[18] X. Hao, L. Liu, Y. Wu, and Q. Sun, "Positive solutions for nonlinear $n$ th-order singular eigenvalue problem with nonlocal conditions," Nonlinear Analysis. Theory, Methods E Applications, vol. 73, no. 6, pp. 1653-1662, 2010.

[19] S. Zhang, "Positive solutions to singular boundary value problem for nonlinear fractional differential equation," Computers $\mathcal{E}$ Mathematics with Applications, vol. 59, no. 3, pp. 1300-1309, 2010.

[20] C. S. Goodrich, "Existence of a positive solution to a class of fractional differential equations," Applied Mathematics Letters, vol. 23, no. 9, pp. 1050-1055, 2010.

[21] M. Rehman and R. A. Khan, "Existence and uniqueness of solutions for multi-point boundary value problems for fractional differential equations," Applied Mathematics Letters, vol. 23, no. 9, pp. 10381044, 2010.

[22] Y. Wang, L. Liu, and Y. Wu, "Positive solutions for a nonlocal fractional differential equation," Nonlinear Analysis. Theory, Methods \& Applications, vol. 74, no. 11, pp. 3599-3605, 2011.

[23] X. Zhang and Y. Han, "Existence and uniqueness of positive solutions for higher order nonlocal fractional differential equations," Applied Mathematics Letters, vol. 25, no. 3, pp. 555-560, 2012.

[24] R. Aris, Introduction to the Analysis of Chemical Reactors, Prentice Hall, Englewood Cliffs, NJ, USA, 1965.

[25] X. Zhang and L. Liu, "Positive solutions of superlinear semipositone singular Dirichlet boundary value problems," Journal of Mathematical Analysis and Applications, vol. 316, no. 2, pp. 525-537, 2006.

[26] Y. Wang, L. Liu, and Y. Wu, "Positive solutions for a class of fractional boundary value problem with changing sign nonlinearity," Nonlinear Analysis. Theory, Methods \& Applications, vol. 74, no. 17, pp. 6434-6441, 2011.

[27] D. J. Guo and V. Lakshmikantham, Nonlinear Problems in Abstract Cones, Academic Press, New York, NY, USA, 1988. 


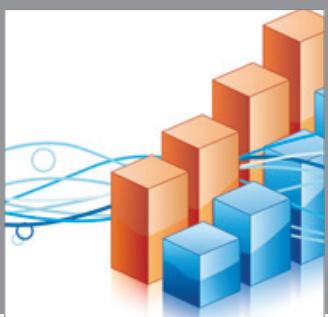

Advances in

Operations Research

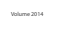

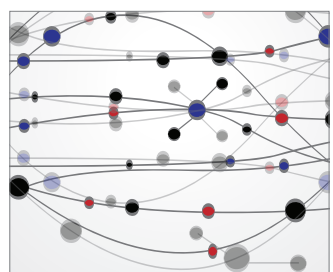

\section{The Scientific} World Journal
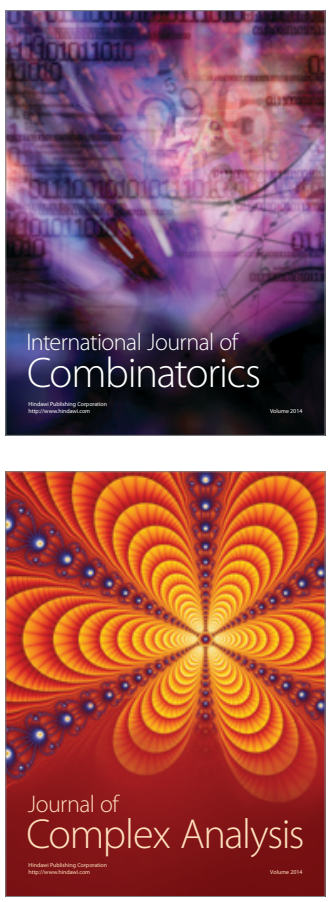

International Journal of

Mathematics and

Mathematical

Sciences
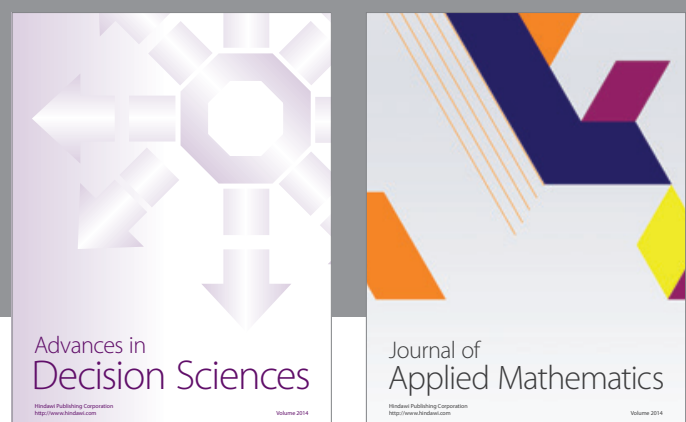

Journal of

Applied Mathematics
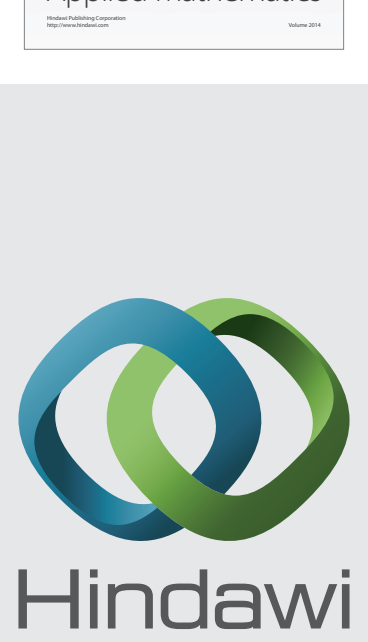

Submit your manuscripts at http://www.hindawi.com
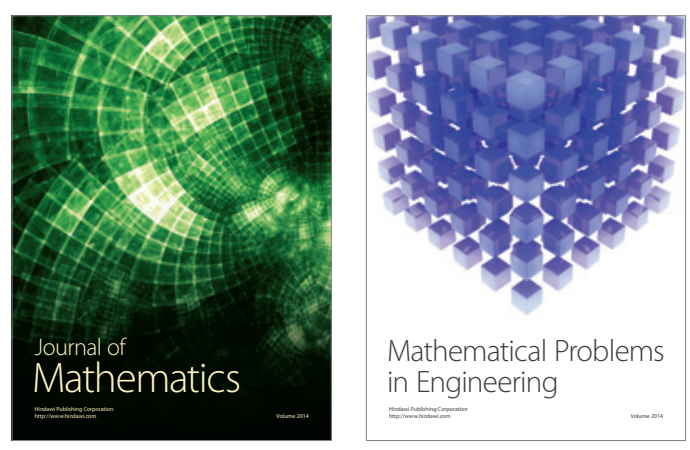

Mathematical Problems in Engineering
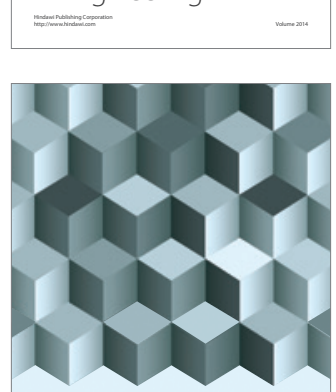

Journal of

Function Spaces
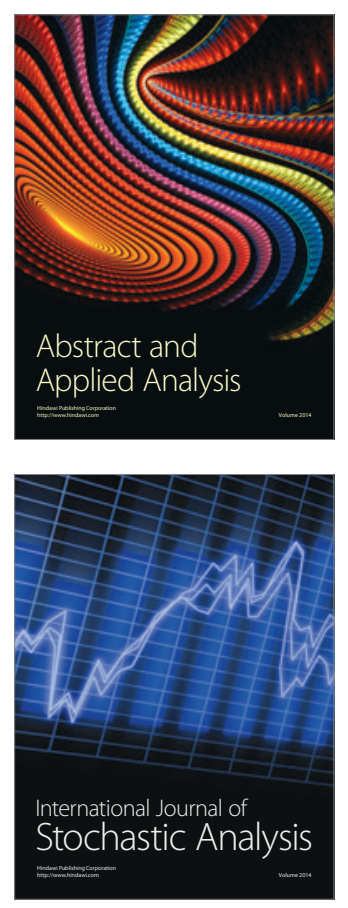

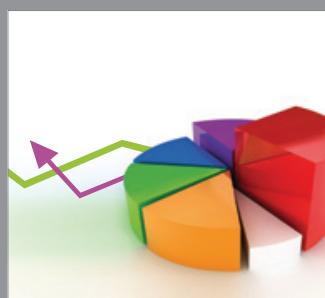

ournal of

Probability and Statistics

Promensencen
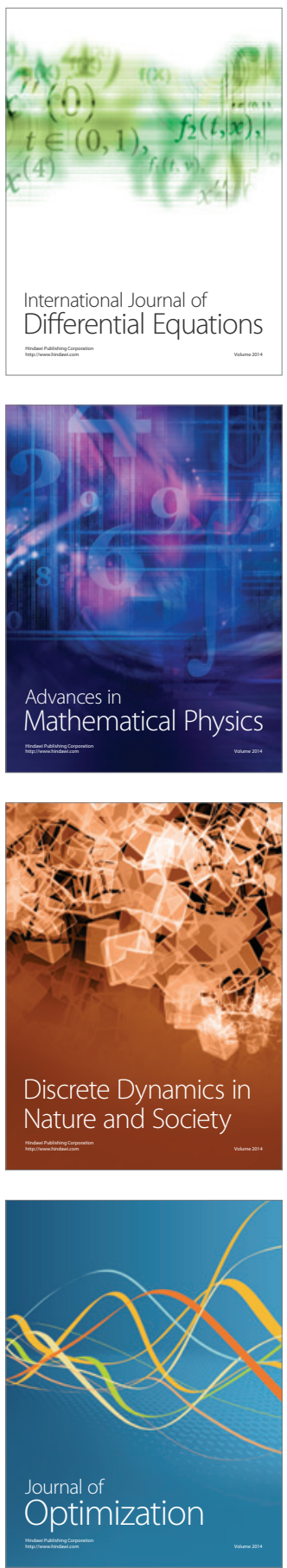